\title{
Polypharmacy Among Headache Patients: A Cross-Sectional Study
}

\author{
Anna Ferrari ${ }^{1}$ (1) $\cdot$ Carlo Baraldi $^{2}$ (1) $\cdot$ Manuela Licata $^{3}$ (I) $\cdot$ Cecilia Rustichelli $^{4}$ (1)
}

Published online: 11 May 2018

(c) The Author(s) 2018

\begin{abstract}
Background Polypharmacy can appropriately treat multiple chronic conditions, but it can also increase potential harm. Polypharmacy information for primary headaches is minimal, despite drugs being the main tools to manage headaches.

Objective The aim was to evaluate the prevalence, characteristics and risk factors of polypharmacy in patients with
\end{abstract}

Electronic supplementary material The online version of this article (https://doi.org/10.1007/s40263-018-0522-8) contains supplementary material, which is available to authorized users.

Anna Ferrari

anna.ferrari@unimore.it

Carlo Baraldi

infocarlobaraldi@gmail.com

Manuela Licata

manuela.licata@unimore.it

Cecilia Rustichelli

cecilia.rustichelli@unimore.it

1 Unit of Medical Toxicology, Department of Diagnostic, Clinical and Public Health Medicine, Headache and Drug Abuse Centre, University of Modena and Reggio Emilia, Via del Pozzo, 71, 41124 Modena, Italy

2 School of Medical Toxicology, University of Modena and Reggio Emilia, Via del Pozzo, 71, 41124 Modena, Italy

3 Forensic Toxicology Laboratory, Department of Diagnostic Medicine, Department of Diagnostic, Clinical and Public Health Medicine, University of Modena and Reggio Emilia, Via del Pozzo, 71, Modena, Italy

4 Department of Life Sciences, University of Modena and Reggio Emilia, via G. Campi, 103, 41125 Modena, Italy primary headaches and examine whether these variables differ between episodic and chronic headache patients.

Methods We analysed polypharmacy (simultaneous use of five or more medications), medication type, comorbidity, and risk factors in 300 patients (mean age $42.81 \pm 13.21$ years) with primary headaches, divided into episodic and chronic, afferent to a headache centre.

Results Patients took an average of 4.37 medications. Polypharmacy was common in $40.7 \%$ of patients, and among chronic patients, it reached $58.8 \%$. Most patients used medications (mainly nonsteroidal anti-inflammatory drugs; $73.5 \%$ ) to treat acute headaches, and $30.4 \%$ of episodic and $64.7 \%$ of chronic sufferers underwent prophylactic treatment $(P<0.0001)$, mostly using antidepressants $(77.3 \%)$. Up to $76.7 \%$ of the cohort was taking other medications, primarily for acid-related disorders $(21.7 \%)$. Comorbidities were present in $59.7 \%$ of the cohort. Variables significantly associated with polypharmacy were comorbidities, prophylactic treatment, and triptans $(P<0.001)$.

Conclusions Patients with primary headaches, mainly young adults, are exposed to high polypharmacy, comparable to that of the elderly. Because increased numbers of drugs increase the risk of adverse reactions, the many medications concomitantly taken by primary headache sufferers should be frequently reviewed. 


\section{Key Points}

Among young adult patients with primary headaches, especially chronic headaches, polypharmacy is common and comparable to that found among the elderly.

Most polypharmacy is due to prescription drugs that patients take to treat their headaches and only to a lesser extent to those used to treat other comorbidities.

These results suggest that medications should be reviewed regularly in primary headache patients, and to avoid risks, discontinuing ineffective or harmful medications may be necessary.

\section{Introduction}

Polypharmacy is defined as the use of multiple medications by an individual patient [1]. This can be appropriate, or even necessary, when no alternatives exist for treating several comorbidities for which different drugs are required and helpful [2]. Nevertheless, concern is growing regarding the risks and complications of polypharmacy [3]. Increases in the number of concomitant medications increases the risk of adverse reactions, which can increase than that of individual drugs. Polypharmacy can also decrease treatment adherence [3, 4] and cause suboptimal health outcomes, wasted medications and a lower quality of life $[1,5,6]$. Polypharmacy has been extensively studied and reported to be prevalent and increasing among the elderly, because of the many subjects with multimorbidities that require multiple medications [4, 7-9].

The prevalence of and complications related to polypharmacy have been evaluated in patients with cancer pain and chronic non-malignant pain $[5,10]$; however, few data $[11,12]$ exist on this issue for primary headaches, despite these disorders being exposed to the possibility of polypharmacy.

Primary headaches are widespread in the general population and prevalent in females [13]. They occur in three main forms: migraine, whose 1-year prevalence is $15 \%$ in the general population; tension-type headache, the most prevalent headache, affecting $60 \%$ of the adult population; and cluster headache, the least frequent type, occurring in less than $1 \%$ of the population [13]. They often remain episodic and improve over time, but may worsen and become chronic and disabling and negatively impact the sufferers' quality of life [14]. Moreover, they are often associated with other medical and psychiatric conditions
[15] that require additional pharmacological treatments. The pathophysiology of primary headaches remains incompletely understood, and no causal and definitive therapies are actually available for these disorders, despite the recent progress in this field [13, 16]. Headache management is mainly based on pharmacological treatments comprising drugs for acute treatment, such as nonsteroidal anti-inflammatory drugs (NSAIDs), triptans, analgesics, muscle relaxants and anti-nausea drugs, and preventive drugs, such as antidepressants, antiepileptics, beta-blocking agents, calcium channel blockers, serotonin antagonists [16] and onabotulinumtoxinA [17]. Acute treatment is always prescribed to headache patients, whereas preventive treatment is recommended in the case of frequent, severe and long-lasting attacks, when symptomatic medications induce only limited effects or are contraindicated, or if the patient's quality of life is seriously affected. Preventive treatment is directed to specifically decrease the frequency of headache attacks and should be taken usually from 3 to 6 months. During the treatment, the patients must often continue taking acute medications for the attack and may need other medications for associated or new-onset illnesses [16].

The objectives of the present study were as follows: (1) to evaluate the prevalence, characteristics and risk factors of polypharmacy, drug utilization and comorbidities in patients with primary headaches afferent to a headache centre; (2) to analyse whether these variables differ between episodic and chronic headache sufferers.

\section{Methods}

\subsection{Study Design and Study Sample}

This cross-sectional, observational study evaluated all drugs regularly taken by 300 primary headache patients consecutively afferent to a headache centre. Patients under 18 years old and suffering from secondary headaches were excluded. The study was approved by the Ethical Committee of the Province of Modena (Prot. n. 3610/C.E., file 126/13). All patients provided written consent to participate in the study and were enrolled from October 1st, 2013 to December 23rd, 2014. The present study is based on unpublished data collected during a research project focused on quantitative determination of triptans and headache prophylactic drugs in hair samples [18-21].

The patients (Table 1) were divided into two groups, namely, (1) episodic headaches (headaches occurring for $<15$ days/month for $>3$ months) and (2) chronic headaches (headaches occurring for $\geq 15$ days/month for $>3$ months), based on the diagnostic criteria of the International Classification of Headache Disorders (ICHD) 
Table 1 Demographic data and comparison between chronic and episodic headache

\begin{tabular}{|c|c|c|c|c|c|}
\hline Variable & Total $n(\%)$ & Episodic headache $n(\%)$ & Chronic headache $n(\%)$ & OR $[95 \% \mathrm{CI}]^{\mathrm{a}}$ & $P$ value $^{\mathrm{b}}$ \\
\hline No. of patients & $300 / 300(100.0)$ & $181 / 300(60.3)$ & $119 / 300(39.7)$ & - & - \\
\hline Inpatients & $73 / 300(24.3)$ & 19/181 (10.5) & $54 / 119(45.4)$ & 7.08 [3.69-13.59] & $<0.0001$ \\
\hline \multicolumn{6}{|l|}{ Outpatients } \\
\hline First visit & $140 / 300(46.7)$ & 102/181 (56.4) & 38/119 (31.9) & $0.36[0.22-0.60]$ & $<0.0001$ \\
\hline Control visit & $87 / 300(29.0)$ & 60/181 (33.1) & $27 / 119(22.7)$ & $0.59[0.35-1.01]$ & 0.0512 \\
\hline Mean age \pm SD (years) & $42.81 \pm 13.21$ & $39.54 \pm 13.08$ & $47.79 \pm 11.32$ & - & $<0.0001$ \\
\hline Range (years) & $18-76$ & $18-76$ & $18-76$ & - & - \\
\hline Females & $286 / 300(95.3)$ & 172/181 (95.0) & $114 / 119(95.8)$ & $1.19[0.39-3.66]$ & 0.7573 \\
\hline \multicolumn{6}{|l|}{ Education } \\
\hline Primary school & $17 / 300(5.7)$ & 11/181 (6.1) & $6 / 119(5.0)$ & $0.82[0.29-2.29]$ & 0.7048 \\
\hline Secondary school & $85 / 300(28.3)$ & $51 / 181(28.2)$ & $34 / 119(28.6)$ & $1.02[0.61-1.70]$ & 0.9409 \\
\hline High school & $124 / 300(41.3)$ & 75/181 (41.4) & 49/119 (41.2) & $0.99[0.62-1.58]$ & 0.9644 \\
\hline University degree & $74 / 300(24.7)$ & 44/181 (24.3) & $30 / 119(25.2)$ & $1.05[0.61-1.79]$ & 0.8597 \\
\hline \multicolumn{6}{|l|}{ Occupation } \\
\hline Employed & 207/300 (69.0) & $119 / 181(65.7)$ & $88 / 119(73.9)$ & $1.48[0.88-2.47]$ & 0.1335 \\
\hline \multicolumn{6}{|l|}{ Ethnicity } \\
\hline Caucasian & 289/300 (96.3) & 171/181 (94.5) & 118/119 (99.2) & $6.90[0.86-55.68]$ & 0.0890 \\
\hline African & $8 / 300(2.7)$ & 7/181 (3.9) & 1/119 (0.8) & $0.21[0.03-1.76]$ & 0.1120 \\
\hline Asian & 3/300 (1.0) & 3/181 (1.7) & 0/119 (0.0) & 0.00 & 0.1590 \\
\hline \multicolumn{6}{|l|}{ Marital status } \\
\hline Married & $167 / 300(55.7)$ & 88/181 (48.6) & 79/119 (66.4) & $2.09[1.28-3.40]$ & 0.0025 \\
\hline
\end{tabular}

Values are $n(\%)$ unless otherwise stated

$C I$ confidence interval, $O R$ odds ratio, $S D$ standard deviation

${ }^{\text {a }}$ chronic vs episodic headache OR

${ }^{\mathrm{b}} p$ value is derived from a Student's $t$ test with equal variances for mean age comparison and from a Chi square test for homogeneity of odds for the others

(3rd edition, beta version) [22]. Group 1 diagnoses were migraine without aura $(70 \%, n=126)$, migraine with aura $(3 \%, n=6)$, migraine with and without aura $(5 \%, n=9)$, and infrequent episodic $(18 \%, n=33)$ and frequent episodic $(4 \%, n=7)$ tension-type headaches. Group 2 diagnoses were chronic migraine $(87 \%, n=103)$ and chronic tension-type headaches $(13 \%, n=16)$. All patients suffering from chronic migraine overused symptomatic medications. Most patients were at least high school graduates, employed, Caucasian, married, and outpatients; female patients represented $95 \%$ of all study subjects. The average age for the entire cohort was $42.81 \pm 13.21$ years. Patients with chronic headaches were significantly older $(47.79 \pm 11.32)$ than those with episodic headaches $(39.54 \pm 13.08)(P<0.0001$, Student's $t$ test $)$. Over $92 \%$ of the total sample were aged $18-60$ years (nearly $70 \%$ of the patients were aged 18-50 years). Episodic headaches were significantly more frequent in patients aged 18-29 years $(P<0.001$, Chi square test $)$, while chronic headaches significantly prevailed in patients aged
51-60 years $(P<0.001$, Chi square test) (see the Electronic Supplementary Material, online resource, ESM_1).

\subsection{Study Variables}

We used a form to collect demographic data from each patient, which included headache diagnosis, comorbidities and a detailed pharmacological history including all medications (dose and route of administration) taken regularly over the previous 3 months. The collected data were assessed based on headache diaries and were discussed between the doctor and patient before being recorded.

\subsection{Data Analysis and Statistics}

The total case studies were descriptively analysed, and groups 1 and 2 were compared in terms of the following variables: demographic data, number and type of medical and psychiatric comorbidities, presence of polypharmacy (simultaneous use of five or more medications), excessive 
medications (simultaneous use of ten or more medications), number, type and pharmacological class of the drugs taken to treat headaches (acute and prophylaxis), and other diseases over the previous 3 months. Moreover, we evaluated the dosage forms taken by the patients for acute treatment and the number of patients using over-the-counter (OTC) and prescription drugs. Medications were coded per the Anatomical Therapeutic Chemical (ATC) classification system. Finally, we performed a multivariate logistic regression analysis to assess the polypharmacy risk factors.

Statistical analysis was conducted using Stata IC13 software. Continuous variables were expressed as the mean \pm standard deviation, and binary variables were expressed as subject count, percentages and odds ratios (ORs). Mean comparison tests were performed using Student's $t$ test for independent data. Binary variables were compared using the Chi square test for homogeneity of odds. To avoid confounding in the multivariate logistic regression analysis, the binary variable indicating the presence or absence of polypharmacy was chosen as the dependent variable, whilst the independent variables were those significantly associated with chronic headaches. Model accuracy was assessed by the receiver-operator characteristic (ROC) curve (see online resource, ESM_2.pdf). $P$ values less than 0.05 were considered significant.

\section{Results}

Headache patients (Table 2) were simultaneously taking an average of more than 4 medications for 3 months, of which at least three were to manage their headaches. Chronic sufferers took significantly more medications than episodic patients $(P<0.0005$, Student's $t$ test). The prevalence of polypharmacy (five or more medications) among the total cohort was $40.7 \%$. In patients with chronic headache, the polypharmacy prevalence was doubled compared with that in the episodic headache patients $(P<0.0001$, Chi square test); excessive polypharmacy (ten or more medications) affected only a minority of the total sample. Most subjects and all chronic patients took acute headache medications. Nearly $65 \%$ of patients with chronic headaches took at least one prophylactic medication, almost twice as many as episodic sufferers $(P<0.0001$, Chi square test). More than three-quarters of the patients were also taking other drugs that were not for headaches.

The most common acute treatment medications (Table 3) were NSAIDs, followed by triptans, and analgesic combinations. Few patients were taking opioids, muscle relaxants or antiemetics. The most frequent active principles were ketoprofen (41\%) among NSAIDs, eletriptan (31\%) among triptans, and tramadol (86\%) among opioids. Patients with chronic headache took triptans $(P<0.0001)$, analgesic combinations $(P<0.05)$ and

Table 2 Characteristics of polypharmacy in the total sample and comparison between chronic and episodic headache patients (some patients took more than one medication at the same time)

\begin{tabular}{|c|c|c|c|c|c|}
\hline Variable & $\begin{array}{l}\text { Total }(n=300), \\
n(\%)\end{array}$ & $\begin{array}{l}\text { Episodic headache } \\
(n=181), n(\%)\end{array}$ & $\begin{array}{l}\text { Chronic headache } \\
(n=119), n(\%)\end{array}$ & OR $[95 \% \mathrm{CI}]^{\mathrm{a}}$ & $P$ value $^{\mathrm{b}}$ \\
\hline \multicolumn{6}{|l|}{ No. of medications taken per patient } \\
\hline Mean \pm SD & $4.37 \pm 2.46$ & $3.64 \pm 2.05$ & $5.49 \pm 2.63$ & & 0.0001 \\
\hline Range & $1-13$ & $1-10$ & $1-13$ & & \\
\hline \multicolumn{6}{|l|}{ No. of headache medications per patient } \\
\hline Mean \pm SD & $3.79 \pm 1.96$ & $2.22 \pm 1.30$ & $3.55 \pm 1.90$ & & $<0.0001$ \\
\hline Range & $0-9$ & $0-7$ & $1-9$ & & \\
\hline \multicolumn{6}{|c|}{ No. of patients taking 5 or more and 10 or more drugs per day } \\
\hline$\geq 5$ medications & $122 / 300(40.7)$ & $52 / 181(28.7)$ & $70 / 119(58.8)$ & $3.54[2.13-5.91]$ & $<0.0001$ \\
\hline$\geq 10$ medications & $9 / 300(3.0)$ & $3 / 181(1.7)$ & $6 / 119(5.0)$ & $3.15[0.77-12.97]$ & 0.0933 \\
\hline \multicolumn{6}{|l|}{ Type of taken medications/supplements } \\
\hline Acute headache medications & $291 / 300(97.0)$ & $172 / 181(95.0)$ & $119 / 119(100.0)$ & $\infty$ & 0.0137 \\
\hline Prophylaxis headache medications & $132 / 300(44.0)$ & $55 / 181(30.4)$ & $77 / 119(64.7)$ & $4.20[2.49-7.09]$ & $<0.0001$ \\
\hline Other medications & $230 / 300(76.7)$ & $133 / 181(73.5)$ & $97 / 119(81.5)$ & $1.59[0.90-2.82]$ & 0.1082 \\
\hline Dietary supplements & $62 / 300(20.7)$ & $38 / 181(21.0)$ & 24/119 (20.2) & $0.95[0.54-1.69]$ & 0.8629 \\
\hline
\end{tabular}

Values are $n(\%)$ unless otherwise stated

$C I$ confidence interval, $O R$ odds ratio, $S D$ standard deviation

${ }^{\mathrm{a}}$ chronic vs episodic headache OR

${ }^{\mathrm{b}} p$ value is derived from a Student's $t$ test with equal variances for the comparison of the number of medications taken daily and from a Chi square test for homogeneity of odds for all the other comparison 
Table 3 Therapeutic classes of medications taken by the 291 patients for acute treatment and comparison between chronic and episodic headache patients (some patients took more than one symptomatic drug)

\begin{tabular}{|c|c|c|c|c|c|}
\hline Therapeutic class & $\begin{array}{l}\text { Total }(n=291) \text {, } \\
n(\%)\end{array}$ & $\begin{array}{l}\text { Episodic headache } \\
(n=172), n(\%)\end{array}$ & $\begin{array}{l}\text { Chronic headache } \\
(n=119), n(\%)\end{array}$ & OR $[95 \% \mathrm{CI}]^{\mathrm{a}}$ & $P$ value $^{\mathrm{b}}$ \\
\hline NSAIDs & 214/291 (73.5) & $133 / 172(77.3)$ & $81 / 119(68.1)$ & $0.63[0.37-1.06]$ & 0.0789 \\
\hline Triptans & $143 / 291(49.1)$ & $65 / 172(37.8)$ & $78 / 119(65.5)$ & $3.13[1.55-5.20]$ & $<0.0001$ \\
\hline Analgesic combinations & 63/291 (21.6) & 29/172 (16.9) & $34 / 119(28.6)$ & $1.97[1.12-3.49]$ & 0.0173 \\
\hline Opioids & $13 / 291(4.5)$ & $2 / 172(1.2)$ & $11 / 119(9.2)$ & 8.66 [1.82-41.09] & 0.0011 \\
\hline Muscle relaxants & $9 / 291(3.1)$ & $3 / 172(1.7)$ & $6 / 119(5.0)$ & $2.99[0.73-12.31]$ & 0.1107 \\
\hline Antiemetics & 5/291 (1.7) & $1 / 172(0.6)$ & 4/119 (3.4) & $5.95[0.65-54.77]$ & 0.0733 \\
\hline
\end{tabular}

CI confidence interval, $O R$ odds ratio, NSAID nonsteroidal anti-inflammatory drug

${ }^{a}$ chronic vs episodic headache OR

${ }^{\mathrm{b}} p$ value is derived from a Chi square test for homogeneity of odds

Table 4 Dosage forms of medications taken by the 291 patients for acute treatment and comparison between chronic and episodic headache patients (some patients took more than one symptomatic drug of more than one dosage form)

\begin{tabular}{|c|c|c|c|c|c|}
\hline Dosage form & $\begin{array}{l}\text { Total }(n=291), \\
n(\%)\end{array}$ & $\begin{array}{l}\text { Episodic headache } \\
(n=172), n(\%)\end{array}$ & $\begin{array}{l}\text { Chronic headache } \\
(n=119), n(\%)\end{array}$ & OR $[95 \% \mathrm{CI}]^{\mathrm{a}}$ & $P$ value $^{\mathrm{b}}$ \\
\hline Oral & 274/291 (94.2) & 161/172 (93.6) & 113/119 (95.0) & $1.29[0.46-3.59]$ & 0.6290 \\
\hline Intramuscolar & $59 / 291(20.3)$ & $31 / 172(18.0)$ & $28 / 119(23.5)$ & $1.40[0.79-2.49]$ & 0.2515 \\
\hline Subcutaneous & $31 / 291(10.7)$ & 13/172 (7.6) & 18/119 (15.1) & $2.18[1.02-4.67]$ & 0.0400 \\
\hline Rectal & 26/291 (8.9) & $12 / 172(7.0)$ & $14 / 119$ (11.8) & $1.78[0.79-4.01]$ & 0.1599 \\
\hline Nasal spray & $6 / 291(2.1)$ & 3/172 (1.7) & $3 / 119(2.5)$ & $1.46[0.29-7.37]$ & 0.6472 \\
\hline
\end{tabular}

CI confidence interval, $O R$ odds ratio

${ }^{\mathrm{a}}$ chronic vs episodic headache OR

${ }^{\mathrm{b}} p$ value is derived from a Chi square test for homogeneity of odds

opioids $(P<0.005)$ (Chi square tests) significantly more than patients with episodic headache. A minority of the sample $(n=75 / 300,25 \%)$ used only OTCs, with no statistically significant differences between patients with chronic headaches $(n=28 / 119,23.5 \%)$ and those with episodic headaches $(n=47 / 181,25.97 \%)$. Most patients (Table 4) took acute treatments orally, and subcutaneous administration was used more by chronic headache sufferers than by episodic headache sufferers $(P<0.05$, Chi square test).
The most commonly used prophylactic drugs (Table 5) were antidepressants and antiepileptics. The most prescribed active principles were amitriptyline (51\%) among antidepressants and topiramate (57\%) among antiepileptics. The percentage of chronic patients treated with antiepileptic drugs was double that of episodic headache patients $(P<0.05$, Chi square test). A majority of patients (Table 6), and more patients with episodic headaches than with chronic headaches $(P<0.05$, Chi square test $)$, used only one medication for acute treatment. Patients with

Table 5 Classes of medications taken by the 132 patients for prophylactic treatment and comparison between chronic and episodic headache patients (some patients took more than one preventive drug)

\begin{tabular}{|c|c|c|c|c|c|}
\hline Therapeutic class & $\begin{array}{l}\text { Total }(n=132), \\
n(\%)\end{array}$ & $\begin{array}{l}\text { Episodic headache } \\
(n=55), n(\%)\end{array}$ & $\begin{array}{l}\text { Chronic headache } \\
(n=77), n(\%)\end{array}$ & OR $[95 \% \mathrm{CI}]^{\mathrm{a}}$ & $P$ value $^{\mathrm{b}}$ \\
\hline Antidepressants & 102/132 (77.3) & $41 / 55(74.6)$ & $61 / 77$ (79.2) & $1.30[0.57-2.97]$ & 0.5290 \\
\hline Antiepileptics & $29 / 132(22.0)$ & $7 / 55(12.7)$ & 22/77 (28.6) & $2.74[1.06-7.13]$ & 0.0308 \\
\hline Beta-blocking agents & $12 / 132(9.1)$ & $5 / 55(9.1)$ & $7 / 77(9.1)$ & $1.00[0.30-3.35]$ & 1.0000 \\
\hline Calcium channel blockers & 9/132 (6.8) & $5 / 55(9.1)$ & $4 / 77(5.2)$ & $0.55[0.14-2.16]$ & 0.3831 \\
\hline
\end{tabular}

CI confidence interval, $O R$ odds ratio

${ }^{\mathrm{a}}$ chronic vs episodic headache OR

${ }^{\mathrm{b}} p$ value is derived from a Chi square test for homogeneity of odds 
Table 6 Number of different active principles for headache treatment taken concurrently in the total sample and comparison between chronic and episodic headache patients (some patients took both acute and prophylactic medications)

\begin{tabular}{|c|c|c|c|c|c|}
\hline $\begin{array}{l}\text { No. of medications taken } \\
\text { for headache treatment }\end{array}$ & $\begin{array}{l}\text { Total }(n=300) \\
n(\%)\end{array}$ & $\begin{array}{l}\text { Episodic headache } \\
(n=181), n(\%)\end{array}$ & $\begin{array}{l}\text { Chronic headache } \\
(n=119), n(\%)\end{array}$ & OR $[95 \% \mathrm{CI}]^{\mathrm{a}}$ & $P$ value $^{\mathrm{b}}$ \\
\hline \multicolumn{6}{|l|}{ For acute treatment } \\
\hline 1 & $109 / 300(36.3)$ & 76/181 (42.0) & $33 / 119$ (27.7) & $0.53[0.32-0.88]$ & 0.0122 \\
\hline 2 & 78/300 (26.0) & 47/181 (26.0) & $31 / 119(26.0)$ & $1.00[0.59-1.70]$ & 0.9871 \\
\hline 3 & $59 / 300(19.7)$ & 33/181 (18.2) & 26/119 (21.8) & $1.25[0.70-2.23]$ & 0.4415 \\
\hline 4 & 27/300 (9.0) & 11/181 (6.1) & $16 / 119(13.4)$ & $2.40[1.06-5.42]$ & 0.0294 \\
\hline 5 & $13 / 300(4.3)$ & $5 / 181(2.8)$ & $8 / 119(6.7)$ & $2.54[0.80-8.01]$ & 0.0999 \\
\hline 6 & $1 / 300(0.3)$ & 0/181 (0) & $1 / 119(0.8)$ & $\infty$ & 0.2175 \\
\hline 7 & $4 / 300(1.3)$ & 0/181 (0) & 4/119 (3.4) & $\infty$ & 0.0132 \\
\hline \multicolumn{6}{|l|}{ For prophylactic treatment } \\
\hline 1 & $95 / 300(31.7)$ & $48 / 181(26.5)$ & $47 / 119(39.5)$ & $1.81[1.10-2.98]$ & 0.0183 \\
\hline 2 & 29/300 (9.7) & $5 / 181(2.8)$ & $24 / 119(20.2)$ & 8.89 [3.14-25.19] & $<0.0001$ \\
\hline 3 & $8 / 300(2.7)$ & 2/181 (1.1) & $6 / 119(5.0)$ & $4.75[1.03-24.30]$ & 0.0587 \\
\hline
\end{tabular}

$C I$ confidence interval, $O R$ odds ratio

achronic vs episodic headache OR

${ }^{\mathrm{b}} p$ value is derived from a Chi square test for homogeneity of odds

Table 7 Other medications [the Anatomical Therapeutic Chemical (ATC) classification, 2nd level, therapeutic subgroup] taken by the 230 patients and comparison between chronic and episodic headache patients (some patients took more than one symptomatic drug)

\begin{tabular}{|c|c|c|c|c|c|}
\hline Other medications (ATC classification) & $\begin{array}{l}\text { Total }(n=230), \\
n(\%)\end{array}$ & $\begin{array}{l}\text { Episodic headache } \\
(n=133), n(\%)\end{array}$ & $\begin{array}{l}\text { Chronic headache } \\
(n=97), n(\%)\end{array}$ & OR $[95 \% \mathrm{CI}]^{\mathrm{a}}$ & $P$ value \\
\hline Drugs for acid related disorders & $50 / 230(21.7)$ & $17 / 133(12.8)$ & 33/97 (34.0) & $3.52[1.78-6.97]$ & 0.0001 \\
\hline Psycholeptics & $46 / 230(20.0)$ & $17 / 133(12.8)$ & 29/97 (29.9) & $2.91[1.46-5.78]$ & 0.0014 \\
\hline Thyroid therapy & $43 / 230(18.7)$ & 26/133 (19.5) & $17 / 97(17.5)$ & $0.87[0.44-1.72]$ & 0.6982 \\
\hline Anti-inflammatory and antirheumatic products & $42 / 230(18.3)$ & $23 / 133(17.3)$ & 19/97 (19.6) & $1.16[0.59-2.29]$ & 0.6572 \\
\hline Antihypertensives & $39 / 230(17.0)$ & $17 / 133(12.8)$ & 22/97 (22.7) & $2.00[1.02-4.05]$ & 0.0487 \\
\hline Sex hormones and modulators of the genital system & $37 / 230(16.1)$ & $25 / 133(18.8)$ & $12 / 97(12.4)$ & $0.61[0.29-1.29]$ & 0.1912 \\
\hline Psychoanaleptics & $26 / 230(11.3)$ & $13 / 133(9.8)$ & $13 / 97(13.4)$ & $1.43[0.63-3.25]$ & 0.3919 \\
\hline Antibacterial for systemic use & $20 / 230(8.7)$ & $13 / 133(9.8)$ & $7 / 97(7.2)$ & $0.72[0.27-1.88]$ & 0.4957 \\
\hline Lipid modifying agents & $14 / 230(6.1)$ & $5 / 133(3.8)$ & 9/97 (9.3) & $2.62[0.84-8.16]$ & 0.0845 \\
\hline Antihistamines for systemic use & $13 / 230(5.7)$ & $10 / 133(7.5)$ & 3/97 (3.1) & $0.39[0.1-1.48]$ & 0.1521 \\
\hline Drugs for functional gastrointestinal disorders & $12 / 230(5.2)$ & $6 / 133(4.5)$ & $6 / 97(6.2)$ & $1.40[0.43-4.48]$ & 0.5737 \\
\hline Corticosteroids for systemic use & $11 / 230(4.8)$ & $5 / 133(3.8)$ & $6 / 97(6.2)$ & $1.69[0.50-5.73]$ & 0.3955 \\
\hline Analgesics & $9 / 230(3.9)$ & $5 / 133(3.8)$ & 4/97 (4.1) & $1.10[0.29-4.22]$ & 0.8883 \\
\hline Drugs for obstructive airway diseases & $7 / 230(3.0)$ & $6 / 133(4.5)$ & $1 / 97(1.0)$ & $0.22[0.03-1.89]$ & 0.1300 \\
\hline Muscle relaxants & $6 / 230(2.6)$ & $3 / 133(2.3)$ & 3/97 (3.1) & $1.38[0.27-7.03]$ & 0.6947 \\
\hline Antiepileptics & $3 / 230(1.3)$ & $1 / 133(0.8)$ & 2/97 (2.1) & $2.78[0.13-16.09]$ & 0.8754 \\
\hline Drugs for treatment of bone diseases & $3 / 230(1.3)$ & $1 / 133(0.8)$ & 2/97 (2.1) & $2.78[0.13-16.09]$ & 0.8754 \\
\hline Immunosuppressants & $1 / 230(0.4)$ & $0 / 133(0.0)$ & 1/97 (1.0) & $\infty$ & 0.2416 \\
\hline
\end{tabular}

CI confidence interval, $O R$ odds ratio

${ }^{\mathrm{a}}$ chronic vs episodic headache OR

${ }^{\mathrm{b}} p$ value is derived from a Chi square test for homogeneity of odds

chronic headache used four medications for acute treatment $(P<0.05$, Chi square test $)$ and two medications for prophylaxis $(P<0.0001$, Chi square test), significantly more than those with episodic headaches.
The first three classes of other medications (Table 7) taken were, in order of frequency, drugs for acid-related disorders (the most used were proton-pump inhibitors, $82 \%$ ), psycholeptics (the most used were benzodiazepines, 
Table 8 Medical and/or psychiatric comorbidities in the 300 primary headache patients and comparison between chronic and episodic headache patients

\begin{tabular}{|c|c|c|c|c|c|}
\hline Number of comorbidities & Total $(n=300), n(\%)$ & $\begin{array}{l}\text { Episodic headache } \\
(n=181), n(\%)\end{array}$ & $\begin{array}{l}\text { Chronic headache } \\
(n=119), n(\%)\end{array}$ & OR $[95 \% \mathrm{CI}]^{\mathrm{a}}$ & $P$ value $^{\mathrm{b}}$ \\
\hline At least 1 & $179 / 300(59.7)$ & 98/181 (54.1) & $81 / 119(68.1)$ & $1.81[1.11-2.94]$ & 0.0164 \\
\hline 1 & $107 / 300(35.7)$ & 68/181 (37.6) & $39 / 119(32.8)$ & $0.81[0.50-1.32]$ & 0.3970 \\
\hline 2 & $52 / 300(17.3)$ & 22/181 (12.2) & $30 / 119$ (25.2) & $2.44[1.31-4.52]$ & 0.0035 \\
\hline$>2$ & $20 / 300(6.7)$ & 8/181 (4.4) & 12/119 (10.1) & $2.43[0.95-6.17]$ & 0.0548 \\
\hline
\end{tabular}

CI confidence interval, $O R$ odds ratio

achronic vs episodic headache OR

${ }^{\mathrm{b}} p$ value is derived from a Chi square test for homogeneity of odds

Table 9 Distribution by type of disorder in the 179 patients with at least one comorbidity and comparison between chronic and episodic headache patients (some patients suffered from more than one comorbidity at the same time)

\begin{tabular}{|c|c|c|c|c|c|}
\hline Comorbidity type & $\begin{array}{l}\text { Total }(n=179), \\
n(\%)\end{array}$ & $\begin{array}{l}\text { Episodic headache } \\
(n=98), n(\%)\end{array}$ & $\begin{array}{l}\text { Chronic headache } \\
(n=81), n(\%)\end{array}$ & OR $[95 \% \mathrm{CI}]^{\mathrm{a}}$ & $P$ value ${ }^{\mathrm{b}}$ \\
\hline Gastrointestinal & $55 / 179(30.7)$ & 21/98 (21.4) & $34 / 81(42.0)$ & $2.65[1.35-5.20]$ & 0.0031 \\
\hline Endocrinological & $51 / 179(28.5)$ & 31/98 (31.6) & 20/81 (24.7) & $0.71[0.36-1.38]$ & 0.3072 \\
\hline Rheumatological & $51 / 179(28.5)$ & $19 / 98(19.4)$ & $32 / 81(39.5)$ & $2.72[1.36-5.41]$ & 0.0031 \\
\hline Psychiatric & $40 / 179(22.3)$ & 18/98 (18.4) & $22 / 81(27.2)$ & $1.66[0.81-3.38]$ & 0.1610 \\
\hline Cardiological & 32/179 (17.9) & $14 / 98(14.3)$ & 18/81 (22.2) & $1.71[0.79-3.73]$ & 0.1690 \\
\hline Respiratory & $17 / 179(9.5)$ & 15/98 (15.3) & $2 / 81(2.5)$ & $0.14[0.03-0.66]$ & 0.0036 \\
\hline Urological & $12 / 179(6.7)$ & 7/98 (7.1) & $5 / 81(6.2)$ & $0.86[0.26-2.81]$ & 0.7967 \\
\hline Haematological & $11 / 179(6.1)$ & 7/98 (7.1) & 4/81 (4.9) & $0.68[0.19-2.41]$ & 0.5421 \\
\hline Obstetric-gynaecological & 6/179 (3.4) & $5 / 98(5.1)$ & $1 / 81(1.2)$ & $0.23[0.03-2.07]$ & 0.1536 \\
\hline Neurological & 1/179 (0.6) & 0/98 (0.0) & $1 / 81(1.2)$ & $\infty$ & 0.2714 \\
\hline
\end{tabular}

$C I$ confidence interval, $O R$ odds ratio

${ }^{\mathrm{a}}$ chronic vs episodic headache OR

${ }^{\mathrm{b}} p$ value is derived from a Chi square test for homogeneity of odds

92\%) and thyroid therapy drugs (the most used was levothyroxine, $91 \%)$. Chronic headache patients took drugs for acid-related disorders $(P<0.0005)$, psycholeptics $(P<0.005)$, and antihypertensives $(P<0.05)$ (Chi square test) more than episodic headache patients. Overall, $64 \%$ of the 300 patients $(n=192)$ were being treated with central nervous system (CNS) drugs, with chronic patients being significantly more prevalent $(n=96 / 119,80.67 \%)$ than episodic patients $(n=96 / 181,53.04 \%) \quad(P<0.0001$, $\mathrm{OR}=3.70$ [2.10-6.50], Chi square test).

Nearly $60 \%$ of the sample (Table 8 ) suffered from at least one comorbidity in addition to headaches. Patients with chronic headaches had significantly more comorbidities than those with episodic headaches, with at least one $(P<0.05)$ and two comorbidities $(P<0.005$, Chi square test). The first three most frequent comorbidities (Table 9$)$ were gastrointestinal (gastritis being the most common, $64 \%$ ), endocrinological (thyroiditis being the most common, $78 \%$ ) and rheumatological disorders (fibromyalgia being the most common, 61\%). Patients with chronic headache suffered from gastrointestinal and rheumatological disorders more often than those with episodic headaches $(P<0.005$, Chi square test), while patients with episodic headaches suffered from respiratory disorders $(P<0.005$, Chi square test $)$ significantly more than those with chronic headaches. The variables significantly associated with polypharmacy (Table 10) were prophylactic treatment, triptans $(P<0.001)$ or other analgesic combination $(P<0.005)$ for acute treatment, and at least one comorbidity $(P<0.001$, Chi square test).

\section{Discussion}

Our results indicate that polypharmacy is a significant issue among primary headache patients. On average, these patients (Table 2) took 4.37 medications together for at least three months, and $40.7 \%$ of the sample simultaneously used five or more medications (polypharmacy). These values were (1) higher than those detected among 
Table 10 Multivariate logistic regression analysis for risk factors of polypharmacy among the 300 primary headache patients

\begin{tabular}{lll}
\hline Variable & OR $^{\mathrm{a}}$ & $P$ value $^{\mathrm{b}}$ \\
\hline Mean age of the patients & -0.026 to 0.023 & 0.911 \\
Inpatients & -0.649 to 0.798 & 0.839 \\
Married/cohabiting & -1.027 to 0.174 & 0.164 \\
Chronic headache & -0.128 to 1.141 & 0.118 \\
Prophylaxis treatment & 0.684 to 1.953 & $<0.001$ \\
Triptans for acute treatment & 0.563 to 1.783 & $<0.001$ \\
Analgesic combination for acute treatment & 0.553 to 2.014 & 0.001 \\
Opioid for acute treatment & -0.035 to 3.685 & 0.055 \\
At least one comorbidity & 1.106 to 2.400 & $<0.001$ \\
\hline$C I$ confidence interval, $O R$ odds ratio & & \\
${ }^{\mathrm{a} O R}$ of polypharmacy & & \\
${ }^{\mathrm{b}} p$ value is derived from a Chi square test for homogeneity of odds &
\end{tabular}

fibromyalgia patients, where polypharmacy was defined as simultaneous intake of three or more medications, which affected $40 \%$ of patients [23]; (2) lower than those of European pain and cancer patients who took an average of 7.8 medications, with over $25 \%$ using ten or more medications [24]; and (3) comparable to those found in the elderly, as approximately $30-40 \%$ of those aged over 65 years are treated with five or more medications [8], and those aged over 80 years have a polypharmacy prevalence of $57.7 \%$ [6]. Notably, our sample was primarily young adult women who were married and working. Here, polypharmacy was mainly due to the medications that patients took to treat their headaches, possibly to perform their daily activities despite the headache. Chronic headache patients took even more medications. Polypharmacy doubled from $28.7 \%$ for patients with episodic headache to $58.8 \%$ for patients with chronic headache $(P<0.0001)$. Overall, these drugs were appropriate as per the guidelines for treating primary headaches [16]. The most used drug classes for acute treatment (Table 3) were NSAIDs $(73.5 \%)$, followed by triptans $(49.1 \%)$. The oral route of administration (Table 4) was preferred by $94.2 \%$ of the sample. Subcutaneous formulations, which allow rapid drug absorption even with nausea and/or vomiting [25], were used more by patients with chronic headaches $(15.1 \%)$ than by those with episodic headaches $(7.6 \%)$ $(P<0.05)$. The guidelines emphasize the importance of prophylactic treatment to reduce headache frequency [16]; however, current prophylactic drugs have limited efficacy. In clinical trials, they decreased the frequency of attacks by $50 \%$ or more in only $55-66 \%$ of patients [26]. In clinical practice, their efficacy was even lower. Of patients with chronic headache, $64.7 \%$ had been taking prophylactic drugs for 3 months, but continued to suffer from chronic headaches. The most common prophylactic drugs were among those at the first level of recommendation per the guidelines [16], which were (Table 5) antidepressants
(77.3\%, the most used was amitriptyline 51\%) and antiepileptics $(22 \%$, the most used was topiramate, $57 \%$ ).

The treatment of pain often requires combinations of various drugs that act with different mechanisms to achieve a synergistic effect [10]. In addition, patients with disabling headaches often consult many specialists looking for a cause and a cure by frequently receiving combinations of prescription medications [12]. This approach generates increased exposure to potential harm [10]. In our study (Table 6), many patients simultaneously used several medications together, both for acute treatment $(60.6 \%$ used at least two) and for prophylaxis (12.4\% used at least two). In the general population, patients with headache often self-treat their condition with OTC analgesics [27-29]. Instead, in our sample of patients who were referred to a headache centre, self-medication with prescription drugs was more common than with OTCs, which involved only $25 \%$ of the sample.

Some of our patients' polypharmacy treated disorders other than headaches. Medical and psychiatric comorbidities are common among pain patients and represent predisposing factors to polypharmacy [30, 31]. In our study (Table 7), the majority of patients $(n=230 / 300,76.7 \%)$ were taking other medications, and 59.7\% $(n=179 / 300)$ suffered (Table 8 ) from at least one comorbidity. The four most used classes of other medications (Table 7) were drugs for acid-related disorders, psycholeptics, thyroid therapy drugs and anti-inflammatory and antirheumatic agents. Consistent with these classes, the most frequent comorbidities (Table 9) were gastrointestinal disorders, followed by endocrinological, rheumatological and psychiatric disorders. Endocrinological disorders were almost exclusively thyroid diseases. The Italian region where the study was conducted (Emilia-Romagna) is characterized by an important iodine deficiency that affects its inhabitants' thyroid health, regardless of headaches [32]. Insomnia is very common among patients with primary headaches [33], 
especially those with migraines [34]. Furthermore, depression is the most frequent comorbidity with chronic pain [31]. Likely due to these disorders, $20 \%$ and $11.3 \%$ of patients consumed psycholeptics and psychoanaleptics, respectively. However, the overall use of CNS medications reached $64 \%$, significantly higher than the $35 \%$ seen in the elderly [35]. Polypharmacy was higher in subjects with chronic headaches. Moreover, $68.1 \%$ had comorbidities and took drugs for acid-related disorders $(P<0.0001)$, psycholeptics $\quad(P<0.005)$ and antihypertensives $(P<0.05)$ significantly more than those with episodic headaches.

Another part of our patients' polypharmacy appeared to depend on a prescribing cascade; i.e. from other medications prescribed to treat adverse drug reactions caused by the drugs used to treat the headaches and comorbidities. For example, the high use of NSAIDs leads to gastropathy as its most common side effect [36]; thus, it is unsurprising that the most employed concomitant medications were for treating acid-related disorders. In addition, the medications used to treat comorbidities may in turn have caused headache as a side effect, thereby increasing the patient's suffering. In fact, headache is one of the most frequent reactions induced by numerous drugs belonging to a large variety of therapeutic classes, and it occurs at therapeutic dose levels. Drugs for the cardiovascular system and in particular antihypertensive agents such as angiotensinconverting-enzyme (ACE) inhibitors, sartans and calcium channel blockers are among those that most frequently cause headache as an adverse reaction [37]. Seventeen per cent of our sample (Table 7) were taking antihypertensive drugs of this type. With the NSAIDs, CNS complaints are less common than gastrointestinal side effects. However, headache, dizziness and drowsiness have been reported during clinical trials with almost all NSAIDs [37]. These drugs were used for disorders other than headache for $18.3 \%$ of the sample. Proton-pump inhibitors have also been associated with headache as a common adverse effect [38]. In our sample, proton-pump inhibitors were the most used class of drugs for acid-related disorders, taken by $21.7 \%$ of patients. Finally, headache is a common side effect of various classes of CNS drugs, some taken by our patients, such as sedative-hypnotic agents (particularly benzodiazepines) and antidepressants (primarily specific inhibitors of serotonin re-uptake such as fluoxetine, paroxetine and sertraline) [38]. In general, headache as an adverse reaction has no specific features and can simulate a primary headache; it can be very difficult to be recognized and diagnosed in a patient who already suffers from headaches. However, it may have the potential to aggravate the primary headache [38].

The most common causes of polypharmacy in the studied patients appeared as a result of the drugs taken for headache management, followed by drugs used for treating comorbidities, and finally, drugs taken for the treatment of side effects from previous medication. We cannot exclude the fact that some agents which cause headache as a side effect may modify or worsen concomitant conditions such as insomnia, anxiety and depression, making them resistant to treatments and ultimately prompting further intake of appropriate medication in an attempt to manage these additional effects. Instead of resolving the headache, this cycle of drug intake may lead to chronicization and overuse of medications, potentially causing further complications.

Lastly, when two or more drugs are co-administered, there is always the possibility of drug-drug interactions, either kinetic or dynamic. The most common mechanisms of kinetic interactions are represented by the induction or inhibition of enzymes of the cytochrome P450 (CYP) system. This system plays an important role in the disposition of a large number of drugs, including those used for primary headache treatment $[39,40]$. The risk of having clinically significant pharmacokinetic drug-drug interactions seems to be limited in patients with low frequency headaches, but could be higher in chronic headache sufferers [41]. Furthermore, if the interaction involves a drug with a narrow therapeutic window, the consequence may be clinically significant. On the basis of the pharmacological classes most commonly used by our patients, and keeping in mind the high frequency of concomitant rheumatological disorders that concerned $28.5 \%$ of patients, it seems important to recommend avoidance of NSAIDs plus drugs with a narrow therapeutic range (i.e. methotrexate), and to also pay attention to the simultaneous use of substrates and inhibitors of CYP2D6 (i.e. metoclopramide, beta-blocking agents, antidepressants) and of CYP3A4 (i.e. eletriptan, verapamil) [39].

The employment of multiple treatments, often decided on an empirical basis, could be avoided if the precision medicine approach [42] was implemented in the management of primary headache. Unfortunately, there have been no specific genetic or epigenetic abnormalities associated with a particular type of headache to assist with determining optimal treatment [43]. In clinical practice, acute and preventive headache treatments are prescribed on the basis of diagnosis, taking into account the comorbidity of the patient and the safety profile of the drug [16], but without the possibility of efficient and optimal individualized treatment for the patient. Recently, calcitonin generelated peptide (CGRP) has emerged as a key neuropeptide involved in the pathophysiology of migraine, and clinical trials are ongoing on various monoclonal antibodies targeted against CGRP or its receptors, which appear to yield promising results [44]. Perhaps, in the future, these antibodies will allow the management of migraine without polypharmacy, and eliminate the risk of chronic migraine 
progression and the overuse of symptomatic medications [43].

Our study has some limitations that should be noted. It was a cross-sectional study, and the data were derived from a secondary analysis. Polypharmacy is more frequent in females [6], and primary headaches (except for cluster headaches, which were unaccounted for in our sample) prevail among women [45]. Therefore, the female prevalence in our study should have allowed greater insight into polypharmacy in primary headache patients. The results refer to patients afferent to a headache centre and were limited by the definitions used. We adopted the numerical definition of polypharmacy identified as the cut-off that signals the clinician to review the patient's current medications [46, 47]. Moreover, this is the first study that quantified and analysed polypharmacy in primary headache patients.

Polypharmacy may be appropriate and necessary in treating pain patients [10]; however, considering that primary headaches are not life-threatening and recur over time, the intake of so many medications, several of which are psychoactive, should be reviewed frequently in young adult subjects. If the risk-to-benefit ratio is unfavourable, the proper intervention should be to stop medications with poor efficacy that reduce the patients' quality of life (i.e. deprescribing), even if theoretically appropriate [48]. This process is uniquely relevant to headaches for the following reasons: (1) Headaches are the most common adverse reaction to many drugs [38]; therefore, the more medications a patient takes, the more likely it is that part of their headache is due to the drugs. (2) Analgesic overuse withdrawal is considered fundamental in treating chronic headaches; this intervention alone could reduce headache frequency [49]. (3) Some headaches are recognized as "refractory" [50]; in these cases, continuing prophylaxis treatments, often in unprecedented combinations that are ineffective after a proper trial and that cause disturbing side effects even if not serious, would be irrational polypharmacy [51].

\section{Conclusion}

In summary, our results show that the prevalence of polypharmacy in patients suffering from primary headaches is high and comparable to that of the elderly [1, 52]. A twenty-year-old migraine patient, during a control visit to review the medications she was taking, exclaimed, "I take more medications than my grandfather!" However, in patients with primary headaches, polypharmacy seems an inevitable consequence of attempting to manage the headaches; in fact, risk factors associated with this condition (Table 10) were prophylactic treatment, use of triptans and analgesic combinations and comorbidities, but not patient age.

We hope that our work can help clinicians to recognize headache sufferers' probable polypharmacy before prescribing other medications; this may represent a step towards improving current primary headache management.

\section{Compliance with Ethical Standards}

Funding This research received no specific grant from any funding agency in the public, commercial, or not-for-profit sectors. The open access fee was paid by a research grant attributed to Dr Anna Ferrari from the Ministry of Education, University and Research (MIUR) (Italy).

Conflict of interest The authors, Anna Ferrari, Carlo Baraldi, Manuela Licata and Cecilia Rustichelli, declare that they have no conflict of interest.

Ethical Approval The study was approved by the Ethical Provincial Committee of Modena (Prot. n. 3610/C.E., file 126/13) and conducted in compliance with the latest version of the Declaration of Helsinki.

Informed Consent All the patients gave their written consent for their participation in the study.

Open Access This article is distributed under the terms of the Creative Commons Attribution-NonCommercial 4.0 International License (http://creativecommons.org/licenses/by-nc/4.0/), which permits any noncommercial use, distribution, and reproduction in any medium, provided you give appropriate credit to the original author(s) and the source, provide a link to the Creative Commons license, and indicate if changes were made.

\section{References}

1. Kaufman G. Polypharmacy: the challenge for nurses. Nurs Stand. 2016;30(39):52-60.

2. Calderón-Larrañaga A, Gimeno-Feliu LA, González-Rubio F, Poblador-Plou B, Lairla-San José M, Abad-Díez JM, PoncelFalcó A, Prados-Torres A. Polypharmacy patterns: unravelling systematic associations between prescribed medications. PLoS ONE. 2013;8(12):e84967.

3. Dwyer LL, Han B, Woodwell DA, Rechtsteiner EA. Polypharmacy in nursing home residents in the United States: results of the 2004 National Nursing Home Survey. Am J Geriatr Pharmacother. 2010;8(1):63-72.

4. Veehof LJ, Stewart RE, Meyboom-de Jong B, Haaijer-Ruskamp FM. Adverse drug reactions and polypharmacy in the elderly in general practice. Eur J Clin Pharmacol. 1999;55(7):533-6.

5. Berndt S, Maier C, Schütz HW. Polymedication and medication compliance in patients with chronic non-malignant pain. Pain. 1993;52(3):331-9.

6. Wauters M, Elseviers M, Vaes B, Degryse J, Dalleur O, Vander Stichele R, Van Bortel L, Azermai M. Polypharmacy in a Belgian cohort of community-dwelling oldest old $(80+)$. Acta Clin Belg. 2016;71(3):158-66.

7. Aparasu RR, Mort JR, Brandt H. Polypharmacy trends in office visits by the elderly in the United States, 1990 and 2000. Res Soc Adm Pharm. 2005;1(3):446-59. 
8. Jokanovic N, Tan EC, Dooley MJ, Kirkpatrick CM, Elliott RA, Bell JS. Why is polypharmacy increasing in aged care facilities? The views of Australian health care professionals. J Eval Clin Pract. 2016;22(5):677-82.

9. Menzies V, Thacker LR 2nd, Mayer SD, Young AM, Evans S, Barstow L. Polypharmacy, opioid use, and fibromyalgia: a secondary analysis of clinical trial data. Biol Res Nurs. 2017;19(1):97-105.

10. Giummarra MJ, Gibson SJ, Allen AR, Pichler AS, Arnold CA. Polypharmacy and chronic pain: harm exposure is not all about the opioids. Pain Med. 2015;16(3):472-9.

11. Oates LN, Scholz MJ, Hoffert MJ. Polypharmacy in a headache centre population. Headache. 1993;33(8):436-8.

12. Putnam GP, O'Quinn S, Bolden-Watson CP, Davis RL, Gutterman DL, Fox AW. Migraine polypharmacy and the tolerability of sumatriptan: a large-scale, prospective study. Cephalalgia. 1999;19(7):668-75.

13. Stovner LJ, Andree C. Prevalence of headache in Europe: a review for the Eurolight project. $J$ Headache Pain. 2010;11(4):289-99.

14. Linde M, Gustavsson A, Stovner LJ, Steiner TJ, Barré J, Katsarava Z, Lainez JM, Lampl C, Lantéri-Minet M, Rastenyte D, Ruiz de la Torre E, Tassorelli C, Andrée C. The cost of headache disorders in Europe: the Eurolight project. Eur $\mathrm{J}$ Neurol. 2012;19:703-11.

15. Buse DC, Manack A, Serrano D, Turkel C, Lipton RB. Sociodemographic and comorbidity profiles of chronic migraine and episodic migraine sufferers. J Neurol Neurosurg Psychiatry. 2010;81:428-32.

16. Sarchielli P, Granella F, Prudenzano MP, Pini LA, Guidetti V, Bono G, Pinessi L, Alessandri M, Antonaci F, Fanciullacci M, Ferrari A, Guazzelli M, Nappi G, Sances G, Sandrini G, Savi L, Tassorelli C, Zanchin G. Italian guidelines for primary headaches: 2012 revised version. J Headache Pain. 2012;13(2):S31-70.

17. Frampton JE, Silberstein S. OnabotulinumtoxinA: a review in the prevention of chronic migraine. Drugs. 2018;78(5):589-600.

18. Ferrari A, Baraldi C, Licata M, Vandelli D, Marchesi F, Palazzoli F, Verri P, Rustichelli C, Giuliani E, Silingardi E. Hair analysis for detection of triptans occasionally used or overused by migraine patients-a pilot study. Eur J Clin Pharmacol. 2016;72(9):1075-81.

19. Licata M, Rustichelli C, Palazzoli F, Ferrari A, Baraldi C, Vandelli D, Verri P, Marchesi F, Silingardi E. Hair testing in clinical setting: simultaneous determination of 50 psychoactive drugs and metabolites in headache patients by LC tandem MS. J Pharm Biomed Anal. 2016;126:14-25.

20. Vandelli D, Palazzoli F, Verri P, Rustichelli C, Marchesi F, Ferrari A, Baraldi C, Giuliani E, Licata M, Silingardi E. Development and validation of a liquid chromatography-tandem mass spectrometric assay for quantitative analyses of triptans in hair. J Chromatogr B Analyt Technol Biomed Life Sci. 2016;1017-1018:136-44.

21. Ferrari A, Licata M, Rustichelli C, Baraldi C, Vandelli D, Marchesi F, Palazzoli F, Verri P, Silingardi E. Monitoring of adherence to headache treatments by means of hair analysis. Eur $\mathbf{J}$ Clin Pharmacol. 2017;73(2):197-203.

22. Headache Classification Committee of the International Headache Society (IHS). The international classification of headache disorders, $3^{\text {rd }}$ edition (beta version). Cephalalgia. 2013;33(9):629-808.

23. Vincent A, Whipple MO, McAllister SJ, Aleman KM, St Sauver JL. A cross-sectional assessment of the prevalence of multiple chronic conditions and medication use in a sample of communitydwelling adults with fibromyalgia in Olmsted County, Minnesota. BMJ Open. 2015;5(3):e006681.
24. Kotlinska-Lemieszek A, Paulsen O, Kaasa S, Klepstad P. Polypharmacy in patients with advanced cancer and pain: a European cross-sectional study of 2282 patients. J Pain Symptom Manage. 2014;48(6):1145-59.

25. Ferrari A, Pinetti D, Bertolini A, Coccia C, Sternieri E. Interindividual variability of oral sumatriptan pharmacokinetics and of clinical response in migraine patients. Eur J Clin Pharmacol. 2008;64(5):489-95.

26. Bigal ME, Krymchantowski AV, Rapoport AM. New developments in migraine prophylaxis. Expert Opin Pharmacother. 2003;4(4):433-43.

27. Mehuys E, Paemeleire K, Crombez G, Adriaens E, Van Hees T, Demarche S, Christiaens T, Van Bortel L, Van Tongelen I, Remon JP, Boussery K. The heterogeneity of headache patients who self-medicate: a cluster analysis approach. Pain. 2016;157(79):1464-71.

28. Katsarava Z, Mania M, Lampl C, Herberhold J, Steiner TJ. Poor medical care for people with migraine in Europe-evidence from the Eurolight study. J Headache Pain. 2018;19(1):10.

29. Steiner TJ, Stovner LJ, Vos T, Jensen R, Katsarava Z. Migraine is first cause of disability in under $50 \mathrm{~s}$ : will health politicians now take notice? J Headache Pain. 2018;19(1):17.

30. Bingefors K, Isacson D. Epidemiology, co-morbidity, and impact on health-related quality of life of self-reported headache and musculoskeletal pain-a gender perspective. Eur $J$ Pain. 2004;8(5):435-50.

31. Fishbain DA. Polypharmacy treatment approaches to the psychiatric and somatic comorbidities found in patients with chronic pain. Am J Phys Med Rehabil. 2005;84(3 Suppl):S56-63.

32. Olivieri A, Di Cosmo C, De Angelis S, Da Cas R, Stacchini P, Pastorelli A, Vitti P. Regional observatories for goiter prevention. The way forward in Italy for iodine. Minerva Med. 2017;108(2):159-68.

33. Uhlig BL, Engstrøm M, Ødegård SS, Hagen KK, Sand T. Headache and insomnia in population-based epidemiological studies. Cephalalgia. 2014;34(10):745-51.

34. Kim J, Cho SJ, Kim WJ, Yang KI, Yun CH, Chu MK. Insomnia in probable migraine: a population-based study. J Headache Pain. 2016;17(1):92.

35. Baftiu A, Feet SA, Larsson PG, Burns ML, Henning O, Sætre E, Molden E, Granas AG, Johannessen SI, Landmark CJ. Utilisation and polypharmacy aspects of antiepileptic drugs in elderly versus younger patients with epilepsy: a pharmacoepidemiological study of CNS-active drugs in Norway, 2004-2015. Epilepsy Res. 2018;139:35-42.

36. Hooper L, Brown TJ, Elliott R, Payne K, Roberts C, Symmons D. The effectiveness of five strategies for the prevention of gastrointestinal toxicity induced by non-steroidal anti-inflammatory drugs: systematic review. BMJ. 2004;329(7472):948.

37. Ferrari A, Spaccapelo L, Gallesi D, Sternieri E. Focus on headache as an adverse reaction to drugs. J Headache Pain. 2009;10(4):235-9.

38. Ferrari A. Headache: one of the most common and troublesome adverse reactions to drugs. Curr Drug Saf. 2006;1(1):43-58.

39. Sternieri E, Coccia CP, Pinetti D, Ferrari A. Pharmacokinetics and interactions of headache medications, part I: introduction, pharmacokinetics, metabolism and acute treatments. Expert Opin Drug Metab Toxicol. 2006;2(6):961-79.

40. Lionetto L, Borro M, Curto M, Capi M, Negro A, Cipolla F, Gentile G, Martelletti P. Choosing the safest acute therapy during chronic migraine prophylactic treatment: pharmacokinetic and pharmacodynamic considerations. Expert Opin Drug Metab Toxicol. 2016;12(4):399-406.

41. De Andrés F, Lionetto L, Curto M, Capi M, Cipolla F, Negro A, Martelletti P. Acute, transitional and long-term cluster headache 
treatment: pharmacokinetic issues. Expert Opin Drug Metab Toxicol. 2016;12(9):1011-20.

42. Aronson SJ, Rehm HL. Building the foundation for genomics in precision medicine. Nature. 2015;526(7573):336-42.

43. Martelletti P. The journey from genetic predisposition to medication overuse headache to its acquisition as sequela of chronic migraine. J Headache Pain. 2018;19(1):2.

44. Holland PR, Goadsby PJ. Targeted CGRP small molecule antagonists for acute migraine therapy. Neurotherapeutics. 2018. https://doi.org/10.1007/s13311-018-0617-4.

45. Lebedeva ER, Kobzeva NR, Gilev DV, Olesen J. Factors associated with primary headache according to diagnosis, sex, and social group. Headache. 2016;56(2):341-56.

46. Gnjidic D, Hilmer SN, Blyth FM, Naganathan V, Waite L, Seibel MJ, McLachlan AJ, Cumming RG, Handelsman DJ, Le Couteur DG. Polypharmacy cutoff and outcomes: five or more medicines were used to identify community-dwelling older men at risk of different adverse outcomes. J Clin Epidemiol. 2012;65(9):989-95.
47. Turner JP, Jamsen KM, Shakib S, Singhal N, Prowse R, Bell JS. Polypharmacy cut-points in older people with cancer: how many medications are too many? Support Care Cancer. 2016;24(4):1831-40.

48. Ailabouni NJ, Nishtala PS, Mangin D, Tordoff JM. Challenges and enablers of deprescribing: a general practitioner perspective. PLoS ONE. 2016;11(4):e0151066.

49. Saper JR, Da Silva AN. Medication overuse headache: history, features, prevention and management strategies. CNS Drugs. 2013;27(11):867-77.

50. Schulman E. Refractory migraine - a review. Headache. 2013;53(4):599-613.

51. Hajjar ER, Cafiero AC, Hanlon JT. Polypharmacy in elderly patients. Am J Geriatr Pharmacother. 2007;5(4):345-51.

52. O’Dwyer M, Peklar J, McCallion P, McCarron M, Henman MC. Factors associated with polypharmacy and excessive polypharmacy in older people with intellectual disability differ from the general population: a cross-sectional observational nationwide study. BMJ Open. 2016;6(4):e010505. 